\title{
Distribution of anaerobic fungi in the digestive tract of cattle and their survival in faeces
}

\author{
David R. Davies, ${ }^{1,2}$ Michael K. Theodorou, ${ }^{1 *}$ Michelle I. G. Lawrence ${ }^{1,2}$ and \\ ANTHONY P. J. TRINCI ${ }^{2}$ \\ ${ }^{1}$ AFRC Institute of Grassland and Environmental Research, Plas Gogerddan, Aberystwyth, Dyfed SY23 3EB, UK \\ ${ }^{2}$ Microbiology Group, Department of Cell and Structural Biology, School of Biological Sciences, Stopford Building, \\ University of Manchester, Manchester M13 9PT, UK
}

(Received 22 October 1992; revised 8 February 1993; accepted 10 February 1993)

\begin{abstract}
A most probable numbers procedure was used to enumerate populations of anaerobic fungi in the digesta and faeces of cattle. Anaerobic fungi were isolated from the rumen, omasum, abomasum, small intestine, caecum, large intestine and faeces. By determining the amount of digesta in each organ of the digestive tract, it was possible to estimate the total population of anaerobic fungi in cattle and make comparisons between populations in different organs. In addition to enumerating anaerobic fungi in freshly collected samples, they were quantified in digesta and faeces which had been dried at ambient temperature and stored in air for up to 9 months. These experiments showed that a higher proportion of the anaerobic fungi present in the hindgut and faeces were able to withstand desiccation than those present within the gastric and pre-gastric organs. Our results support the hypothesis that the life cycle of anaerobic fungi consists of three stages; the motile zoospore, the vegetative thallus and an aero-tolerant survival stage (cyst or resistant zoosporangium).
\end{abstract}

\section{Introduction}

The digestive tract of many herbivores has evolved such that the plant biomass ingested can be degraded in a way which best serves the nutrition of the animal. In this respect, ruminants are particularly dependent on the activity of micro-organisms and it is now accepted that their gut microflora consist of a diverse population of bacteria, protozoa and fungi which can grow under highly anaerobic conditions.

Anaerobic fungi were first isolated from ruminants (cattle and sheep) by Orpin (1975) and Bauchop (1979) and have since been found in the digesta and faeces of numerous other ruminant and monogastric herbivores, including post-gastric fermenters such as the elephant, horse and rhino (Theodorou et al., 1992). Lowe et al. (1987) were the first to isolate anaerobic fungi from the faeces of sheep. These authors obtained isolates from faeces which were morphologically similar to Neocallimastix and Caecomyces species found in the rumen.

\footnotetext{
*Author for correspondence. Tel. (0970) 828255; fax (0970) 828357.
}

Abbreviations: t.f.u., thallus forming units; MPN, most probable number; DM, dry matter.
In further studies, Milne et al. (1989) isolated anaerobic fungi from the faeces of a wide range of herbivores and demonstrated the resistance of these organisms to desiccation by isolating them from faeces that had been dried and stored in air for up to 18 weeks. More recently, Theodorou et al. (1990) developed a most probable numbers (MPN) procedure for the enumeration of anaerobic fungi in rumen digesta and faeces. Using this technique, it was shown that ruminants could contain approximately equivalent numbers of anaerobic fungi (relative to digesta or faecal dry matter) in fresh faeces as in their rumen digesta. Unlike anaerobic fungi in faeces, anaerobic fungi in rumen digesta fail to survive drying (Milne et al., 1989; Theodorou et al., 1990).

Representatives of four of the recognized genera of anaerobic fungi, Neocallimastix, Piromyces, Caecomyces and Orpinomyces, commonly found in rumen digesta, have been obtained from both fresh and air-dried faeces (Lowe et al., 1987; Milne et al., 1989; Wubah et al., $1991 a$; D. R. Davies, M. K. Theodorou, A. P. J. Trinci \& B. B. Nielsen, unpublished results). Where anaerobic fungi have been found in faeces, particularly in the faeces of wild herbivores, their presence in the digestive tract is usually assumed. Although anaerobic fungi in the rumen have been of considerable interest since their discovery, 
the ability of these obligately fermentative organisms to survive in other organs of the digestive tract and in faeces has been given relatively little attention.

One hypothesis to explain the large populations of resistant anaerobic fungi found in faeces is that they can produce a resting stage (a survival structure) in their life cycle (Theodorou et al., 1990). In the work reported here, fresh and dried samples of faeces and digesta from each organ of the digestive tract of cattle were investigated for the presence of anaerobic fungi. The results obtained provide the first quantification of anaerobic fungi in the entire ruminant digestive tract and are consistent with the hypothesis that anaerobic fungi are able to produce resistant structures.

\section{Methods}

Animals sampled. Entire digestive tract studies, in which anaerobic fungi were enumerated in faeces and in digesta from each of the digestive tract organs, were performed on three groups of animals, each consisting of three animals as follows: (a) lactating Friesian/Holstein dairy cows, where enumerations were performed on fresh digesta and faeces; (b) 8-month-old Friesian/Holstein steers, where enumerations were performed on digesta and faeces prior to and after drying and storage in air for $7 \mathrm{~d}$; and (c) 20-month-old Friesian/Holstein steers, where enumerations were performed on digesta and faeces prior to and after drying and storage in air for up to 9 months. All animals were fed an ad libitum perennial ryegrass (Lolium perenne L.) silage diet with supplements. Dairy cows received per day $3 \mathrm{~kg}$ barley-based concentrates containing $20 \%(\mathrm{w} / \mathrm{w})$ crude protein. Fishmeal was added to the diet of the 8-month-old steers at a rate of $10 \%(w / w)$ dry matter (DM) of the daily silage intake. The diet of the 20 -month-old steers was not supplemented. Water and mineral blocks were freely available to all animals at all times.

Sampling procedure. The alimentary tract was removed intact from the carcass immediately after slaughter and dissected into organs, after tying off the various sections with bailer twine to reduce the likelihood of cross-contamination between organs (particularly between the abomasum and small intestine). Digesta samples (approximately $200-400 \mathrm{~g}$ fresh wt) for fungal enumeration, $\mathrm{DM}$ and $\mathrm{pH}$ determinations were removed from the rumen, omasum, abomasum, small intestine, caecum and large intestine; an approximately equivalent amount of faeces was collected from animals prior to slaughter. The $\mathrm{pH}$ of digesta and faeces was determined using a mobile $\mathrm{pH}$ meter (Philips). Depending on the part of the tract being sampled, the total gut contents from each organ was determined by one of two methods. Either the contents of the entire organ were emptied into a container and weighed directly, or the organ and its contents were weighed, the contents removed and the empty organ weighed after washing. This latter procedure was used for the omasum where it was impractical to remove all of the digesta contents without washing. The time taken between slaughter and fungal enumeration was approximately $1 \mathrm{~h}$ and during this period samples were maintained at about $37^{\circ} \mathrm{C}$.

Enumeration of anaerobic fungi. Anaerobic fungi were enumerated as thallus forming units (t.f.u.) per g digesta or faecal DM, and per organ, using wheat straw as the substrate $[1 \%(\mathrm{w} / \mathrm{v})$ wheat straw ground through a $1 \mathrm{~mm}$ dry mesh screen], as described by Theodorou et al. (1990). Enumerations were performed on $5 \mathrm{~g}$ (fresh wt) quantities of fresh sample or samples which had been dried and kept in air at ambient temperature for up to 9 months. The positive and negative tubes from MPN enumerations, using three replicates per dilution and 10 -fold dilutions, were quantified according to the method of Ross (1987).

The enumeration medium, designated Medium $\mathrm{C}$, was based on that of Orpin (1977). Medium C contained $\left(l^{-1}\right)$ : salts solution I $(150 \mathrm{ml})$, salts solution II $(150 \mathrm{ml})$, centrifuged (clarified) rumen fluid $(150 \mathrm{ml})$, Yeast extract (Oxoid) $(2.5 \mathrm{~g})$, Trypticase peptone (Becton and Dickinson) $(10.0 \mathrm{~g}), \mathrm{NaHCO}_{3}(6.0 \mathrm{~g})$, resazurin solution $(0 \cdot 1 \%, \mathrm{w} / \mathrm{v})(1.0 \mathrm{ml})$, L-cysteine hydrochloride $(1.0 \mathrm{~g})$. Salts solution I contained $\mathrm{K}_{2} \mathrm{HPO}_{4}$ $\left(3.0 \mathrm{~g} \mathrm{l}^{-1}\right)$ in distilled water. Salts solution II contained each of the following components $\left(\mathrm{g} \mathrm{l}^{-1}\right)$ dissolved in distilled water in the following order: $\mathrm{KH}_{2} \mathrm{PO}_{4}(3 \cdot 0),\left(\mathrm{NH}_{4}\right)_{2} \mathrm{SO}_{4}(6 \cdot 0), \mathrm{NaCl}(6 \cdot 0), \mathrm{MgSO}_{4} \cdot 7 \mathrm{H}_{2} \mathrm{O}(0 \cdot 6)$, $\mathrm{CaCl}_{2} \cdot 2 \mathrm{H}_{2} \mathrm{O}(0 \cdot 6)$.

With the exception of L-cysteine hydrochloride, each component was added in the amount indicated and the medium made up to 11 with distilled water. The L-cysteine hydrochloride was added after the medium was gassed for $1 \mathrm{~h}$ with oxygen-free $\mathrm{CO}_{2}$. Aliquots $(45 \mathrm{ml})$ were dispensed into $60 \mathrm{ml}$ serum bottles using anaerobic techniques and autoclaved at $121^{\circ} \mathrm{C}$ for $15 \mathrm{~min}$. Prior to use in the MPN procedure, $1 \mathrm{ml}$ of chloramphenicol solution was added to each bottle of Medium C. The antibiotic solution contained $5 \mathrm{mg}$ chloramphenicol $\mathrm{ml}^{-1}$ dissolved in $50 \%(\mathrm{v} / \mathrm{v})$ aqueous ethanol.

\section{Results}

\section{$p H$ and dry matter of digesta and faeces}

The $\mathrm{pH}$ of digesta in pre-gastric (reticulo-rumen and omasum) and post-gastric (caecum and large intestines) organs and the small intestines, and of faeces of all animals, ranged from neutral to slightly acidic (Table 1). In the abomasum, which is the site of gastric digestion, digesta contents were more acidic, with mean $\mathrm{pH}$ values ranging from 4.9 for dairy cows to 3.3 for both growing and mature steers (Table 1).

Within each group of animals, the total amount of digesta DM was highest in the rumen, ranging from $3881 \mathrm{~g}$ in growing steers to $9656 \mathrm{~g}$ in lactating dairy cows (Table 1). Other organs contained much less DM, ranging from $151 \mathrm{~g}$ in the large intestine of growing steers to $1077 \mathrm{~g}$ in the small intestine of dairy cows (Table 1). Distribution of DM throughout the digestive tract was remarkably consistent across all animals with approximately $84.0 \%$ contained within the pre-gastric organs, approximately $10.6 \%$ in the abomasum and small intestines and only about $5.4 \%$ in the post-gastric organs. Values for total digesta DM content were used subsequently, together with data obtained from the MPN procedure, to calculate fungal t.f.u. per organ and fungal t.f.u. per $\mathrm{g}$ digesta or faecal DM.

\section{Fungal populations in digesta and faeces}

Fungal populations (enumerated as t.f.u. per g DM and t.f.u. per organ) in digesta and faeces from dairy cows, growing steers and mature steers are shown in Table 2. Except for the small intestine, where anaerobic fungi were cultured from two out of three dairy cows and two 
Table 1. pH and dry matter content in each organ of the digestive tract and in faeces of cattle

Each value represents the mean of three observations \pm SD. The animals were: $(a)$ Friesian/Holstein dairy cows of mean live weight $570 \mathrm{~kg}$ and fed ad libitum silage diet with $3 \mathrm{~kg}$ barley-based concentrates per d; $(b)$ 8 -month-old Friesian/Holstein steers of mean live weight $244 \mathrm{~kg}$ and fed an ad libitum silage diet, with fishmeal added at a rate of $10 \%$ dry matter of daily silage intake; (c) 20-month-old Friesian/Holstein steers of mean live weight $497 \mathrm{~kg}$ and fed an ad libitum silage diet without supplements. Total dry matter (DM) was determined by collecting the entire digesta from each organ, obtaining a fresh matter weight and then adjusting for the percentage dry matter of representative samples.

\begin{tabular}{|c|c|c|c|c|c|c|}
\hline \multirow[b]{2}{*}{ Organ } & \multicolumn{2}{|c|}{ Dairy cow } & \multicolumn{2}{|c|}{ Growing steer } & \multicolumn{2}{|c|}{ Mature steer } \\
\hline & $\mathrm{pH}$ & $\begin{array}{c}\text { Total DM } \\
\text { per organ }(\mathrm{g})\end{array}$ & $\mathrm{pH}$ & $\begin{array}{c}\text { Total DM } \\
\text { per organ }(\mathrm{g})\end{array}$ & $\mathrm{pH}$ & $\begin{array}{c}\text { Total DM } \\
\text { per organ }(\mathrm{g})\end{array}$ \\
\hline Reticulo-rumen & $5 \cdot 7 \pm 0.64$ & $9656 \pm 1085$ & $6 \cdot 7 \pm 0 \cdot 13$ & $3881 \pm 584$ & $6 \cdot 5 \pm 0.21$ & $6339 \pm 476$ \\
\hline Omasum & $6 \cdot 3 \pm 0 \cdot 32$ & $463 \pm 244$ & $6 \cdot 6 \pm 0.17$ & $329 \pm 97$ & $6.6 \pm 0.12$ & $1054 \pm 143$ \\
\hline Abomasum & $4 \cdot 9 \pm 0 \cdot 44$ & $290 \pm 261$ & $3.3 \pm 0.47$ & $152 \pm 10$ & $3 \cdot 3 \pm 0 \cdot 10$ & $252 \pm 27$ \\
\hline Small intestine & $7 \cdot 0 \pm 0.34$ & $1077 \pm 367$ & $5.7 \pm 0.04$ & $443 \pm 66$ & $6.7 \pm 0.00$ & $500 \pm 39$ \\
\hline Caecum & $6.7 \pm 0.06$ & $269 \pm 114$ & $7 \cdot 0 \pm 0 \cdot 12$ & $169 \pm 5$ & $6.7 \pm 0.06$ & $179 \pm 70$ \\
\hline Large intestine & $6.6 \pm 0.09$ & $290 \pm 85$ & $6.9 \pm 0.05$ & $151 \pm 32$ & $6.7 \pm 0.00$ & $277 \pm 69$ \\
\hline Faeces & $6 \cdot 5 \pm 0 \cdot 17$ & $\mathrm{NA}$ & $6.9 \pm 0.07$ & $\overline{\mathrm{NA}}$ & $6.7 \pm 0.00$ & $\overline{\mathrm{NA}}$ \\
\hline
\end{tabular}

NA, Not applicable.

Table 2. Fungal t.f.u. per g dry matter (DM) and t.f.u. per organ for digesta and faeces from cattle

Each value represents the mean of three observations. The MPN enumerations used 10 -fold dilutions and three replicates per dilution. Total t.f.u. per organ were calculated from the total digesta dry matter per organ (see Table 1) and the t.f.u. per g DM for that organ. The lower $95 \%$ confidence limits for the enumerations were approximately $4.0-5.0$ fold lower than the values presented in the Table. With the exception of the caecum in growing and mature steers where the upper $95 \%$ confidence limits were $5 \cdot 4$ - and $6 \cdot 2$-fold higher than the values presented in the Table, the upper limits were approximately 3.0-4.0-fold higher than the values presented. NA, Not applicable.

\begin{tabular}{|c|c|c|c|c|c|c|}
\hline \multirow[b]{2}{*}{ Organ } & \multicolumn{3}{|c|}{ t.f.u. $(\mathrm{g} \mathrm{DM})^{-1}$} & \multicolumn{3}{|c|}{ t.f.u. per organ } \\
\hline & $\begin{array}{l}\text { Dairy } \\
\text { cows }\end{array}$ & $\begin{array}{c}\text { Growing } \\
\text { steers }\end{array}$ & $\begin{array}{l}\text { Mature } \\
\text { steers }\end{array}$ & $\begin{array}{l}\text { Dairy } \\
\text { cows }\end{array}$ & $\begin{array}{c}\text { Growing } \\
\text { steers }\end{array}$ & $\begin{array}{l}\text { Mature } \\
\text { steers }\end{array}$ \\
\hline Reticulo-rumen & $6.03 \times 10^{4}$ & $1.17 \times 10^{5}$ & $2.04 \times 10^{5}$ & $5.82 \times 10^{8}$ & $4.54 \times 10^{8}$ & $1.29 \times 10^{9}$ \\
\hline Omasum & $8.91 \times 10^{4}$ & $1.82 \times 10^{5}$ & $1.20 \times 10^{5}$ & $4.13 \times 10^{7}$ & $5.99 \times 10^{7}$ & $1.26 \times 10^{8}$ \\
\hline Abomasum & $2.69 \times 10^{3}$ & $5.75 \times 10^{3}$ & $3.02 \times 10^{4}$ & $7.80 \times 10^{5}$ & $8.74 \times 10^{5}$ & $7.61 \times 10^{6}$ \\
\hline Small intestine & $8.31 \times 10^{2}$ & $2 \cdot 18 \times 10^{2}$ & $1.29 \times 10^{4}$ & $8.95 \times 10^{5}$ & $9.66 \times 10^{4}$ & $6.45 \times 10^{6}$ \\
\hline Caecum & $7.08 \times 10^{3}$ & $2.88 \times 10^{4}$ & $1.41 \times 10^{4}$ & $1.90 \times 10^{6}$ & $4.87 \times 10^{6}$ & $2.52 \times 10^{6}$ \\
\hline Large intestine & $3.31 \times 10^{3}$ & $4.90 \times 10^{4}$ & $4.27 \times 10^{4}$ & $9.60 \times 10^{5}$ & $7.40 \times 10^{6}$ & $1.18 \times 10^{7}$ \\
\hline Faeces & $6.03 \times 10^{3}$ & $2 \cdot 10 \times 10^{4}$ & $9 \cdot 77 \times 10^{4}$ & NA & NA & NA \\
\hline
\end{tabular}

out of three growing steers, digesta from pre-gastric, gastric and post-gastric organs, and faeces all contained fungal populations. The distribution of anaerobic fungi throughout the digestive tract was similar for each group of animals. When expressed relative to digesta or faecal $\mathrm{DM}$, the largest populations of anaerobic fungi were found associated with digesta contents from the rumen and omasum. These ranged from $6.03 \times 10^{4}$ t.f.u. $(\mathrm{g} \mathrm{DM})^{-1}$ in the rumen digesta of dairy cows to $2.04 \times 10^{5}$ t.f.u. $(\mathrm{g} \mathrm{DM})^{-1}$ in the rumen digesta of mature steers (Table 2). Within each group of animals, fungal populations associated with digesta from the gastric and post-gastric organs, and from faeces were at least an order of magnitude lower than those associated with digesta from the rumen and omasum. In three situations, i.e. (a) digesta from the small intestines of dairy cows, (b) digesta from the abomasum of growing steers, and (c) digesta from the small intestines of growing steers, fungal t.f.u. $(\mathrm{g} \mathrm{DM})^{-1}$ were up to three orders of magnitude lower than those detected in digesta from the comparative pre-gastric organs (Table 2).

When expressed as t.f.u. per organ, fungal populations were highest in the rumen and omasum, accounting for approximately $89 \%$ and $9 \%$ respectively of the anaerobic fungi in the entire digestive tract (Table 2). Fungal populations declined considerably (by up to 1000 -fold) in the abomasum and small intestines and then remained unaltered or increased slightly in the caecum and large intestines (Table 2). Expressed as a percentage of the total, the fungal populations in the 
Table 3. Fungal t.f.u. per $g$ dry matter (DM) and the percentage survival of t.f.u. of the original fresh sample for dried digesta and faeces from growing steers

Each value represents the mean of three observations. The MPN enumerations used 10 -fold dilutions and three replicates per dilution. The animals were 8 -month-old steers with a mean live weight of $244 \mathrm{~kg}$ and were fed an ad libitum silage diet with fishmeal at $10 \%$ of the dry matter of the daily silage intake. Digesta and faeces were dried in air for $7 \mathrm{~d}$ at ambient room temperature in aluminium dishes. The $95 \%$ confidence limits represent the statistically possible lower and upper t.f.u. counts with a $95 \%$ degree of confidence.

\begin{tabular}{lcccc}
\hline \hline & & \multicolumn{2}{c}{$95 \%$ Confidence limits } & \\
\cline { 3 - 4 } \multicolumn{1}{c}{ Organ } & $\begin{array}{c}\text { Fungal t.f.u. } \\
(\mathrm{g} \mathrm{DM})^{-1}\end{array}$ & Lower & Upper & $\begin{array}{c}\text { Percentage of } \\
\text { original sample }\end{array}$ \\
\hline Reticulo-rumen & 4.57 & 1.62 & $1.02 \times 10^{1}$ & 0.004 \\
Omasum & $3.98 \times 10^{3}$ & $8.91 \times 10^{2}$ & $1.45 \times 10^{4}$ & 2.2 \\
Abomasum & $5.89 \times 10^{2}$ & $1.26 \times 10^{2}$ & $2.00 \times 10^{3}$ & 10.2 \\
Small intestine & 0.00 & 0.00 & $3.02 \times 10^{1}$ & 0.0 \\
Caecum & $2.19 \times 10^{3}$ & $4.78 \times 10^{2}$ & $8.91 \times 10^{3}$ & 7.7 \\
Large intestine & $3.31 \times 10^{3}$ & $1.12 \times 10^{3}$ & $1.74 \times 10^{4}$ & 6.8 \\
Faeces & $5.13 \times 10^{3}$ & $1.12 \times 10^{3}$ & $1.74 \times 10^{4}$ & 24.5 \\
\hline \hline
\end{tabular}

hindgut (caecum and large intestines) ranged from $0.5 \%$, in dairy cows, to $1 \cdot 0 \%$, in mature steers, to $2 \cdot 3 \%$, in growing steers, of the population in the entire digestive tract. By summation of fungal t.f.u. in each organ, the mean population of anaerobic fungi in the entire digestive tract of the mature steers was calculated as $1.44 \times 10^{9}$ t.f.u.; populations in growing steers and lactating dairy cows were slightly lower at $5.27 \times 10^{8}$ and $6.28 \times 10^{8}$ t.f.u. per digestive tract, respectively.

\section{Fungal populations in dried digesta and faeces after $7 d$ storage in air}

When samples of digesta and faeces from growing steers were enumerated $7 \mathrm{~d}$ after the onset of drying and storage, all except digesta from the small intestines and two of the three replicate samples from the rumen were found to contain populations of anaerobic fungi. Fungal populations in dried digesta and faeces, expressed as t.f.u. $(\mathrm{g} \mathrm{DM})^{-1}$ and as a percentage of those present in the original fresh samples, are shown in Table 3. Upon drying and storage, fungal populations in rumen digesta declined by 5 orders of magnitude [from $1.17 \times 10^{5}$ to 4.57 t.f.u. $\left(\mathrm{g} \mathrm{DM}^{-1}\right.$ ], representing a mean survival of $0.004 \%$ of the total population originally present (Table 3 ). Thus, if all of the digesta from the rumen had been dried, of the $4.54 \times 10^{8}$ t.f.u. initially present, only $1.82 \times 10^{4}$ would have survived the drying process. Fungal populations in omasal digesta declined by approximately 50 -fold, representing a survival ability of $2 \cdot 2 \%$ of the initial population (Table 3 ). Thus, the fungal population in the omasum was about 550 times more able to survive the drying process than that present in the rumen. Samples of digesta from the abomasum, hindgut organs and from faeces, after drying, had values ranging from $6.8 \%$ to $24.5 \%$ of the original populations (Table 3 ). Thus, the fungi in digesta from these organs and the fungi in faeces were approximately 2000 to 6000 times more able to survive desiccation and storage than those present in rumen digesta, fungi in faeces having the greatest ability to survive.

\section{Fungal populations in dried digesta and faeces after prolonged storage in air}

Samples of digesta and faeces from mature steers were enumerated for anaerobic fungi, both when they were fresh and at various times after drying and storage. All fresh samples contained fungal populations (Table 2, Fig. 1). In all cases when survival occurred, the ultimate size of the surviving population was small in comparison to that present in the fresh sample. However, as in the previous experiment, the proportion of the surviving population increased in digesta from organs remote from the reticulo-rumen and in faeces.

Fungal t.f.u. in digesta from the small intestines, rumen and abomasum declined rapidly after drying such that viable populations were detectable only within the 1st, 7th and 22nd d of the experiment, respectively (Fig. 1). Omasal digesta retained a fungal population of approximately $1 \%$ of that present in the original fresh sample, but viable fungi were not detected after $57 \mathrm{~d}$ storage. Anaerobic fungi in digesta from post-gastric organs and faeces were more tolerant to desiccation and storage than those from the pre-gastric and gastric organs. Thus, the fungi present in caecal digesta were able to survive for up to $109 \mathrm{~d}$, whereas those present in digesta from the large intestines and faeces maintained 


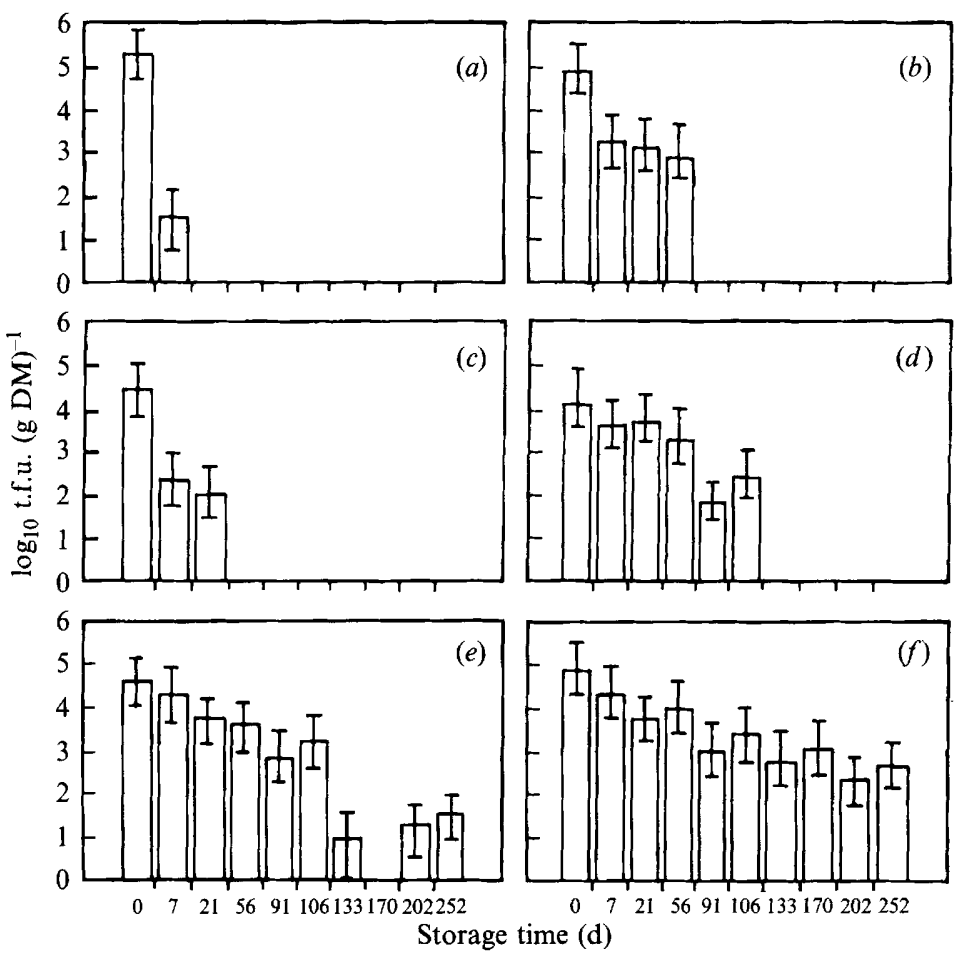

Fig. 1. Fungal populations in fresh and dried digesta and faeces from mature steers, dried in air and stored for up to $252 \mathrm{~d}$. Each value represents the mean of three observations. The MPN enumerations used 10 -fold dilutions and 3 replicates per dilution. The 20 -month-old steers had a mean live weight of $497 \mathrm{~kg}$ and were fed an ad libitum silage diet. Digesta and faeces were dried and stored in air for periods up to $252 \mathrm{~d}$ at ambient room temperature in aluminium dishes. (a) Reticulo-rumen; (b) omasum; (c) abomasum; $(d)$ caecum; $(e)$ large intestine; $(f)$ faeces. Data for the small intestine are not presented as these samples did not have a viable anaerobic fungal population upon drying. Error bars represent the upper and lower $95 \%$ confidence limits of the t.f.u. enumerations. detectable populations for $252 \mathrm{~d}$ of storage, when the experiment was terminated (Fig. 1).

\section{Discussion}

It is now accepted that anaerobic fungi have an important role in ruminal cellulolysis (Theodorou et al., 1989, 1992). However, other than the information presented in this paper, and by Grenet et al. (1989), Milne et al. (1989), Davies et al. (1990) and Theodorou et al. (1990), little is known about fungal populations inhabiting parts of the digestive tract other than the rumen. This study has shown, for the first time, that anaerobic fungi are present throughout the entire digestive tract and provides the first enumeration of anaerobic fungi in these organs. The MPN procedure requires the enumeration tubes to be frequently observed using the light microscope (Theodorou et al., 1990). From these observations the morphological characteristics of numerous fungal isolates, from both fresh and dried samples of digesta and faeces, were determined. Observations were limited to a cursory examination of zoospores and thalli and no attempt was made to segregate different isolates into genera and species. Nonetheless, the examination was sufficiently thorough to enable us to conclude that all samples contained representatives of the monocentric genera, Neocallimastix, Piromyces and Caecomyces. Thallus development was endogenous and the tapering rhizoids of Neocallimastix and Piromyces spp. were extensively branched, whilst those of the Caecomyces were more limited, taking the form of bulbous spherical bodies or holdfasts. Zoospores were uninucleate, spherical, oval or waisted in appearance, with one (Piromyces, Caecomyces) or numerous (Neocallimastix) flagella. Anaerobic fungi belonging to the polycentric genera (e.g. Orpinomyces) were not observed during the course of this study, although they have been isolated from dried ruminant faeces on a separate occasion. For the animals studied, approximately $89 \%$ of the fungal population inhabited the rumen, whereas about $9 \%$ occupied the omasum and the remaining $2 \%$ was distributed between the organs of the midgut and hindgut (Table 2). Thus, the total population of anaerobic fungi in the rumen was considerably larger than that in the omasum and the midgut and hindgut organs. When fungal populations were related to the amount of DM in the sample, t.f.u. values in digesta from the rumen and omasum were approximately equal (Table 2). Compared with the pregastric organs, however, there was a marked decrease in fungal populations in digesta from the abomasum and small intestine, and these lesser populations remained unaltered or increased slightly in the hindgut organs and in faeces (Table 2).

Fungi present in the omasum and lower gut are probably carried out of the rumen on particulate material; this view is also held by Grenet et al. (1989) to explain their observations of the presence of anaerobic fungi in the duodenum and caecum of dairy cows. The dramatic decline in fungal populations in the midgut of 


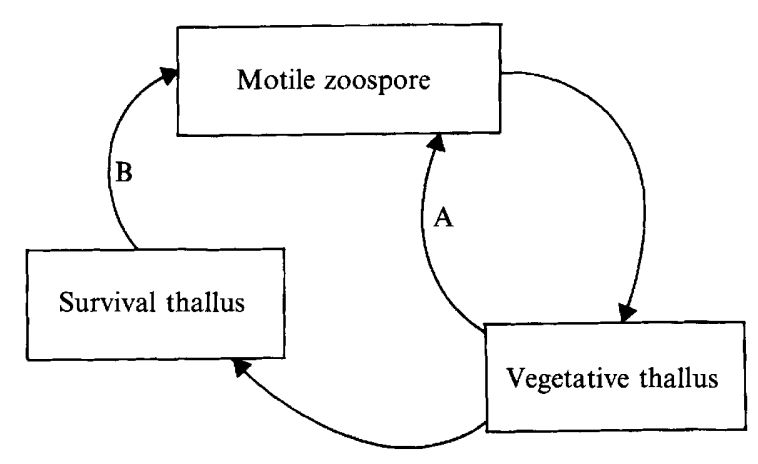

Fig. 2. Diagrammatic representation of the complete life cycle of anaerobic fungi. Cycle $A$ is the vegetative cycle purported to occur in the rumen. Cycle B is the survival cycle which occurs when conditions become unfavourable for vegetative growth.

all animals tested, and subsequent increase in populations in the hindgut of some animals can be explained by the assumptions that $(a)$ many zoospores and immature thalli may be killed during gastric digestion in the abomasum and that $(b)$ some fungal growth and liberation of zoospores continues in the hindgut in much the same way as it does in the rumen.

Of the total population of anaerobic fungi in the digestive tract of growing steers $\left(5 \cdot 27 \times 10^{8}\right)$, only $0.43 \%$ $\left(2.29 \times 10^{6}\right)$ were able to withstand drying in air for $7 \mathrm{~d}$. Of these surviving fungi, the majority $(99.9 \%)$ were located in parts of the digestive tract other than the rumen. Moreover, a much greater percentage ( $24.5 \%$ ) of the fungi present in faeces were able to withstand the drying process than those present in any organ of the digestive tract (Table 3). These observations were confirmed in a similar experiment, in which anaerobic fungi from the digesta and faeces of mature steers were enumerated in both fresh samples and samples at various times after drying and storage. In this case, in addition to increasing survival with distance down the tract fungal populations in digesta from the hindgut and in faeces were able to survive for considerably longer periods than those present in the foregut and midgut regions (Fig. 1). These results serve to illustrate that fungi in the lower tract and in faeces, although lower in numbers, were more able to survive desiccation and exposure to air than those present in the rumen.

To account for our findings, we have amended the generally accepted fungal life cycle by inclusion of an additional survival stage as shown in Fig. 2. Cycle A is the vegetative cycle purported to occur in the rumen. Cycle B is an alternative route which is activated when conditions for vegetative growth become less favourable. We speculate that the survival stage is a thick walled zoosporangium which is formed in a proportion of the anaerobic fungi leaving the rumen. This structure can then germinate when conditions become favourable, either in the hindgut if substrates are available, or after defaecation and re-introduction into a new host animal. Resistant cysts are common amongst aerobic chytrids and have recently been reported in the genus Neocallimastix (Wubah et al., 1991b). These workers identified thick-walled zoosporangia which contained melanin and increased DNA levels, but which failed to germinate in batch culture.

The authors would like to thank Cathy King-Spooner, Mandy Fyrth, Barbara Williams and Lola Isac for their technical assistance. We would also like to thank Malcolm Gibb and Wendy Ivings for the provision of entire digestive tract samples. We thank the Agriculture and Food Research Council and the Science and Engineering Research Council for supporting this work.

\section{References}

BAUCHOP, T. (1979). Rumen anaerobic fungi of cattle and sheep. Applied and Environmental Microbiology 38, 148-158.

Davies, D., Theodorou, M. K. \& Trinci, A. P. J. (1990). Anaerobic fungi in the digestive tract and faeces of growing steers: evidence for a third stage in their life cycle. Fourth International Mycological Congress, Abstracts, IIE-226/3, edited by A. Reisinger \& A. Bresinsky. Regensburg, Germany: Botanical Institute, University of Regensburg.

GrenEt, E., FonTy, G., JAMOT, J. \& BonNEMOY, F. (1989). Influence of diet and monensin on the development of anaerobic fungi in the rumen, duodenum, cecum and feces of cows. Applied and Environmental Microbiology 55, 2360-2364.

Lowe, S. E., Theodorou, M. K. \& TRINCI, A. P. J. (1987). Isolation of anaerobic fungi from saliva and faeces of sheep. Journal of General Microbiology 133, 1829-1834.

Milne, A., Theodorou, M. K., Jordan, M. G. C., King-Spooner, C. \& TrINCI, A. P. J. (1989). Survival of anaerobic fungi in feces, in saliva, and in pure culture. Experimental Mycology 13, 27-37.

ORPIN, C. G. (1975). Studies on the rumen flagellate Neocallimastix frontalis. Journal of General Microbiology 91, 249-262.

OrPIN, C. G. (1977). The rumen flagellate Piromonas communis: its lifehistory and invasion of plant material in the rumen. Journal of General Microbiology 99, 107-117.

Ross, G. J. S. (1987). MLP; Maximum likelihood program. Harpenden: Rothamstead Experimental Station.

Theodorou, M. K., Longland, A. C., DhanoA, M. S., Lowe, S. E. \& TRINCI, A. P. J. (1989). Growth of Neocallimastix sp. strain R1 on Italian ryegrass hay: removal of neutral sugars from plant cell walls. Applied and Environmental Microbiology 55, 1363-1367.

Theodorou, M. K., Gill, M., King-Spooner, C. \& BeEver, D. E. (1990). Enumeration of anaerobic chytridiomycetes as thallus forming units: a novel method for the quantification of fibrolytic fungal populations from the digestive tract ecosystem. Applied and Environmental Microbiology 56, 1073-1078.

Theodorou, M. K., Lowe, S. E. \& TRINCI, A. P. J. (1992). Anaerobic fungi and the rumen ecosystem. In The Fungal Community: It's Organisation and Role in the Ecosystem, 2nd edn, pp. 43-72. Edited by G. C. Carroll \& D. T. Wicklow. New York: Marcel Dekker.

Wubah, D. A., Fuller, M.S. \& AkIN, D. E. (1991a). Isolation of monocentric and polycentric fungi from the rumen and feces of cows in Georgia. Canadian Journal of Botany 69, 1232-1236.

Wubah, D. A., Fuller, M. S. \& Akin, D. E. (1991b). Resistant body formation in Neocallimastix sp., an anaerobic fungus from the rumen of a cow. Mycologia 83, 40-47. 